\title{
Stability of the Regular Hayward Thin-Shell Wormholes
}

\author{
M. Sharif and Saadia Mumtaz \\ Department of Mathematics, University of the Punjab, Quaid-e-Azam Campus, Lahore 54590, Pakistan \\ Correspondence should be addressed to M. Sharif; msharif.math@pu.edu.pk \\ Received 23 May 2016; Revised 1 July 2016; Accepted 10 July 2016 \\ Academic Editor: Luis A. Anchordoqui
}

Copyright ( 2016 M. Sharif and S. Mumtaz. This is an open access article distributed under the Creative Commons Attribution License, which permits unrestricted use, distribution, and reproduction in any medium, provided the original work is properly cited. The publication of this article was funded by $\mathrm{SCOAP}^{3}$.

\begin{abstract}
The aim of this paper is to construct regular Hayward thin-shell wormholes and analyze their stability. We adopt Israel formalism to calculate surface stresses of the shell and check the null and weak energy conditions for the constructed wormholes. It is found that the stress-energy tensor components violate the null and weak energy conditions leading to the presence of exotic matter at the throat. We analyze the attractive and repulsive characteristics of wormholes corresponding to $a^{r}>0$ and $a^{r}<0$, respectively. We also explore stability conditions for the existence of traversable thin-shell wormholes with arbitrarily small amount of fluid describing cosmic expansion. We find that the space-time has nonphysical regions which give rise to event horizon for $0<a_{0}<2.8$ and the wormhole becomes nontraversable producing a black hole. The nonphysical region in the wormhole configuration decreases gradually and vanishes for the Hayward parameter $l=0.9$. It is concluded that the Hayward and Van der Waals quintessence parameters increase the stability of thin-shell wormholes.
\end{abstract}

\section{Introduction}

One of the most interesting attributes of general relativity is the possible existence of hypothetical geometries having nontrivial topological structure. Misner and Wheeler [1] described these topological features of space-time as solutions of the Einstein field equations known as wormholes. A "wormhole" having a tunnel with two ends allows a short way associating distant regions of the universe. Besides the lack of some observational lines of evidence, wormholes are regarded as a part of black holes (BH) family [2]. The simplest example is the Schwarzschild wormhole that connects one part of the universe to another through a bridge. This wormhole is not traversable as it does not allow a twoway communication between two regions of the space-time leading to the contraction of wormhole throat.

Physicists have been motivated by the proposal of Lorentzian traversable wormholes given by Morris and Thorne [3]. In case of traversable wormholes, the wormhole throat is threaded by exotic matter which causes repulsion against the collapse of the wormhole throat. The most distinguishing property of these wormholes is the absence of event horizon which enables observers to traverse freely across the universe. It was shown that a $\mathrm{BH}$ solution with horizons could be converted into wormhole solution by adding some exotic matter which makes the wormhole stable [4]. Traversable wormhole solutions must satisfy the flare-out condition preserving their geometry due to which the wormhole throat remains open. The existence of exotic matter yields the violation of null energy condition (NEC) and weak energy condition (WEC) which is the basic property for traversable wormholes. Null energy condition is the weakest one whose violation gives rise to the violation of WEC and strong energy conditions (SEC). The exotic matter is characterized by stressenergy tensor components determined through Israel thinshell formalism [5].

Thin-shell wormholes belong to one of the wormhole classes in which exotic matter is restricted at the hypersurface. To make sure that the observer does not encounter nonphysical zone of $\mathrm{BH}$, a thin shell strengthens the wormhole provided that it has an exotic matter for its maintenance against gravitational collapse. The physical viability of thinshell wormholes is a debatable issue due to inevitable amount of exotic matter which is an essential ingredient for the existence as well as stability of wormholes. The amount of exotic matter can be quantified by the volume integral 
theorem which is consistent with the concept that a small quantity of exotic matter is needed to support wormhole [6]. Visser [7, 8] developed an elegant cut-and-paste technique to minimize the amount of exotic matter by restricting it at the edges of throat in order to obtain a more viable thin-shell wormhole solution.

It is well known that thin-shell wormholes are of significant importance if they are stable. The stable/unstable wormhole models can be investigated either by applying perturbations or by assuming equation of state (EoS) supporting exotic matter at the wormhole throat. In this context, many authors constructed thin-shell wormholes following Visser's cut-and-paste procedure and discussed their stability. Kim and Lee [9] investigated stability of charged thin-shell wormholes and found that charge affects stability without affecting the space-time itself. Ishak and Lake [10] analyzed stability of spherically symmetric thin-shell wormholes. Lobo and Crawford [11] studied spherically symmetric thin-shell wormholes with cosmological constant $(\Lambda)$ and found that stable solutions exist for positive values of $\Lambda$. Eiroa and Romero [12] studied linearized stability of charged spherical thin-shell wormholes and found that the presence of charge significantly increases the possibility of stable wormhole solutions. Sharif and Azam [13] explored both stable and unstable configurations for spherical thin-shell wormholes. Sharif and Mumtaz analyzed stable wormhole solutions from regular ABG [14] and ABGB [15] space-time in the context of different cosmological models for exotic matter.

It is found that one may construct a traversable wormhole theoretically with arbitrarily small amount of fluid describing cosmic expansion. In order to find any realistic source for exotic matter, different candidates of dark energy have been proposed like tachyon matter [16], family of Chaplygin gas $[17,18]$, phantom energy [19], and quintessence [20]. Eiroa [21] assumed generalized Chaplygin gas to study the dynamics of spherical thin-shell wormholes. Kuhfittig [22] analyzed stability of spherical thin-shell wormholes in the presence of $\Lambda$ and charge by assuming phantom like EoS at the wormhole throat. Sharif and collaborators discussed stability analysis of Reissner-Nordström [23] and Schwarzschild de Sitter as well as anti-de Sitter [24] thin-shell wormholes in the vicinity of generalized cosmic Chaplygin gas (GCCG) and modified cosmic Chaplygin gas (MCCG). Some physical properties of spherical traversable wormholes [25] as well as stability of cylindrical thin-shell wormholes $[26,27]$ have been studied in the context of GCCG, MCCG, and Van der Waals (VDW) quintessence EoS. Recently, Halilsoy et al. [28] discussed stability of thin-shell wormholes from regular Hayward $\mathrm{BH}$ by taking linear, logarithmic, and Chaplygin gas models and found stable solutions for increasing values of Hayward parameter.

This paper is devoted to the construction of thin-shell wormholes from regular Hayward $\mathrm{BH}$ by considering three different models of exotic matter at the throat. The paper is organized as follows. In Section 2, we construct regular Hayward thin-shell wormholes and analyze various physical aspects of these constructed thin-shell wormholes. Section 3 deals with stability formalism of the regular Hayward thinshell wormholes in the vicinity of VDW quintessence EoS and Chaplygin gas models. We find different throat radii numerically and show their expansion or collapse with different values of parameters. Finally, we provide summary of the obtained results in the last section.

\section{Regular Hayward Black Hole and Wormhole Construction}

The static spherically symmetric regular Hayward $\mathrm{BH}$ [29] is given by

$$
\begin{aligned}
d s^{2}= & -F(r) d t^{2}+F^{-1}(r) d r^{2} \\
& +G(r)\left(d \theta^{2}+\sin ^{2} \theta d \phi^{2}\right),
\end{aligned}
$$

where $G(r)=r^{2}$ and $F(r)=1-2 M r^{2} /\left(r^{3}+2 M l^{2}\right)$ and $M$ and $l$ are positive constants. This regular $\mathrm{BH}$ is chosen for thinshell wormhole because a regular system can be constructed from finite energy and its evolution is more acceptable. This reduces to de Sitter $\mathrm{BH}$ for $r \rightarrow 0$, while the metric function for the Schwarzschild $\mathrm{BH}$ is obtained as $r \rightarrow \infty$. Its event horizon is the largest root of the equation

$$
r^{3}-2 M r^{2}+2 M l^{2}=0
$$

This analysis of the roots shows a critical ratio $l / M_{*}=4 / 3 \sqrt{3}$ and radius $r_{*}=\sqrt{3} l$ such that, for $r>0$ and $M<M_{*}$, the given space-time has no event horizon yielding a regular particle solution. The regular Hayward $\mathrm{BH}$ admits a single horizon if $r=r_{*}$ and $M=M_{*}$, which represents a regular extremal BH. At $r=r_{ \pm}$and $M>M_{*}$, the given space-time becomes a regular nonextremal $\mathrm{BH}$ with two event horizons.

We implement the standard cut-and-paste procedure to construct a timelike thin-shell wormhole. In this context, the interior region of the regular Hayward $\mathrm{BH}$ is cut with $r<a$. The two 4D copies are obtained which are glued at the hypersurface $\Sigma^{ \pm}=\Sigma=\{r=a\}$. In fact, this technique treats the hypersurface $\Sigma$ as the minimal surface area called wormhole throat. The exotic matter is concentrated at the hypersurface making the wormhole solution a thin shell. We can take coordinates $\chi^{i}=(\tau, \theta, \phi)$ at the shell. The induced $3 \mathrm{D}$ metric at $\Sigma$ with throat radius $a=a(\tau)$ is defined as

$$
d s^{2}=-d \tau^{2}+a^{2}(\tau)\left(d \theta^{2}+\sin ^{2} \theta d \phi^{2}\right)
$$

where $\tau$ is the proper time on the shell.

This construction requires the fulfillment of flare-out condition by the throat radius $a$; that is, the embedding function $G(r)$ in (1) should satisfy the relation $G^{\prime}(a)=2 a>0$. The thin layer of matter on $\Sigma$ causes the extrinsic curvature discontinuity. In this way, Israel formalism is applied for the dynamical evolution of thin shell which allows matching of 


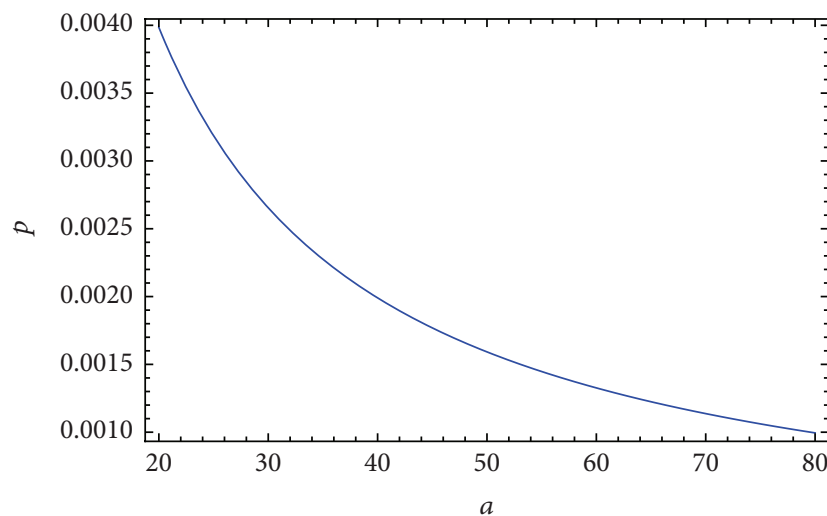

(a)

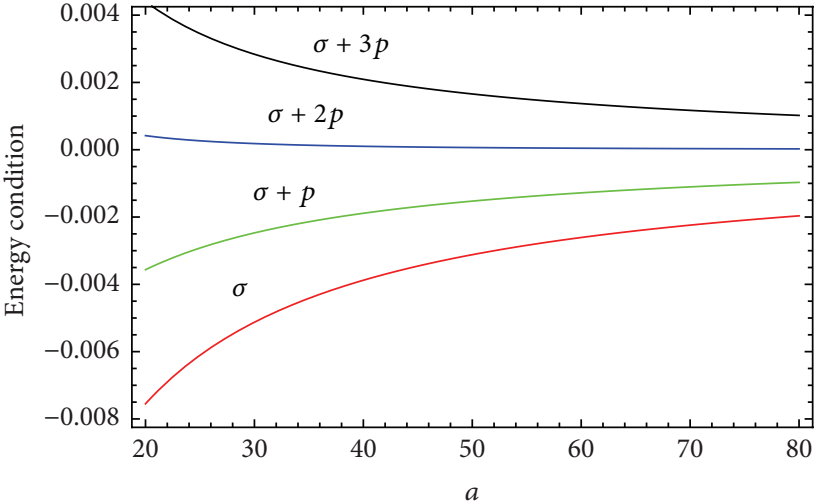

(b)

Figure 1: Plots of $p$ and energy conditions with $M=1$ and $l=0.9$.

two regions of space-time partitioned by $\Sigma$. We find nontrivial components of the extrinsic curvature as

$$
\begin{aligned}
& K_{\tau \tau}^{ \pm}=\mp \frac{F^{\prime}(a)+2 \ddot{a}}{2 \sqrt{F(a)+\dot{a}^{2}}}, \\
& K_{\theta \theta}^{ \pm}= \pm a \sqrt{F(a)+\dot{a}^{2}}, \\
& K_{\phi \phi}^{ \pm}=\alpha^{2} K_{\theta \theta}^{ \pm},
\end{aligned}
$$

where dot and prime stand for $d / d \tau$ and $d / d r$, respectively. To determine surface stresses at the shell, we use Lanczos equations, which are the Einstein equations given by

$$
S_{i j}=\frac{1}{8 \pi}\left\{g_{i j} K-\left[K_{i j}\right]\right\} \text {, }
$$

where $\left[K_{i j}\right]=K_{i j}^{+}-K_{i j}^{-}$and $K=\operatorname{tr}\left[K_{i j}\right]=\left[K_{i}^{i}\right]$. The surface energy-momentum tensor $S_{i j}$ yields the surface energy density $S_{\tau \tau}=\sigma$ and surface pressures $S_{\theta \theta}=p=S_{\phi \phi}$. Solving (4) and (5), the surface stresses are obtained as

$$
\begin{aligned}
& \sigma=-\frac{1}{2 \pi a} \sqrt{F(a)+\dot{a}^{2}}, \\
& p=p_{\theta}=p_{\phi}=\frac{1}{8 \pi} \frac{2 \dot{a}^{2}+2 a \ddot{a}+2 F(a)+a F^{\prime}(a)}{a \sqrt{F(a)+\dot{a}^{2}}} .
\end{aligned}
$$

In order to prevent contraction of wormhole throat, matter distribution of surface energy-momentum tensor must be negative which indicates the existence of exotic matter making the wormhole traversable $[7,8]$. The amount of this matter should be minimized for the sake of viable wormhole solutions. We note from (6) and (7) that $\sigma<0$ and $\sigma+p<0$ showing the violation of NEC and WEC for different values of $M, l$, and $a$. In Figure 1, we plot a graph for pressure showing that pressure is a decreasing function of the throat radius (a), while (b) shows violation of energy conditions associated with regular Hayward thin-shell wormholes.
Now we explore the attractive and repulsive characteristics of the regular Hayward thin-shell wormholes. In this context, we need to compute the observer's four-acceleration

$$
a^{\mu}=u_{; \nu}^{\mu} u^{\nu}
$$

where $u^{\mu}=d x^{\mu} / d \tau=(1 / \sqrt{F(r)}, 0,0,0)$ is the observer's four-velocity. The nonzero four-acceleration component corresponding to the given space-time is calculated as

$$
a^{r}=\Gamma_{t t}^{r}\left(\frac{d t}{d \tau}\right)^{2}=\frac{M r^{4}-4 M^{2} l^{2} r}{\left(r^{3}+2 m l^{2}\right)^{2}},
$$

for which the geodesic equation has the following form:

$$
\frac{d^{2} r}{d \tau^{2}}=-\Gamma_{t t}^{r}\left(\frac{d t}{d \tau}\right)^{2}=-a^{r}
$$

An important condition for traversing through a wormhole implies that an observer should not be dragged away by enormous tidal forces. It is required that the acceleration felt by the observer must not exceed Earth's acceleration. It is worth stressing here that a wormhole will be attractive in nature if its radial acceleration is positive; that is, $a^{r}>0$. This supports the fact that an observer must have outwarddirected radial acceleration $a^{r}$ in order to keep away from being pulled by the wormhole. On the other hand, it will exhibit repulsive characteristics for $a^{r}<0$. In this case, an observer must move with inward-directed radial acceleration to avoid being repelled by the wormhole. The attractive and repulsive characteristics of the regular Hayward thin-shell wormholes are shown in Figure 2.

Some researchers are excited by the possibility of wormholes in reality. It appears feasible to keep the wormhole throat open long enough such that an object can traverse easily through it if the throat is threaded by exotic matter. The total amount of exotic matter is quantified by the integral theorem [6]

$$
\Omega=\int\left[\rho+p_{r}\right] \sqrt{-g} d^{3} x .
$$




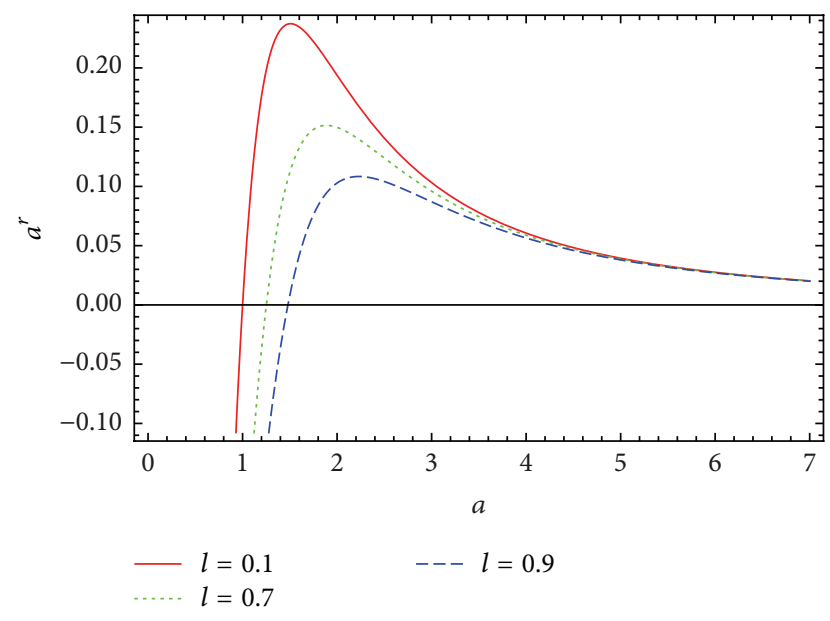

FIGURE 2: Plots of $a^{r}$ with $M=1$ and $l=0.1,0.7,0.9$. The wormhole is attractive for $a^{r}>0$ and repulsive for $a^{r}<0$.

By introducing radial coordinate $R=r-a$, we have

$$
\Omega=\int_{0}^{2 \pi} \int_{0}^{\pi} \int_{-\infty}^{+\infty}\left[\rho+p_{r}\right] \sqrt{-g} d R \sin \theta d \theta d \phi
$$

The wormhole shell, being thin, does not apply any pressure leading to $p_{r}=0$. Using $\rho=\delta(R) \sigma(a)$, we have

$$
\Omega_{a}=\left.\int_{0}^{2 \pi}[\rho \sqrt{-g}]\right|_{r=a} d \phi=2 \pi a \sigma(a) .
$$

Inserting the value of surface energy density $\sigma(a)$, the above expression yields

$$
\Omega_{a}=-\sqrt{\frac{a_{0}^{3}+2 M l^{2}-2 M a_{0}^{2}}{a_{0}^{3}+2 M l^{2}}},
$$

where $a_{0}$ is the wormhole throat radius. It is interesting to note that construction of a traversable wormhole is possible theoretically with vanishing amount of exotic matter. This amount can be made infinitesimally small by choosing exotic fluids explaining cosmic expansion.

\section{Stability of Thin-Shell Wormholes}

Here, we study the formation of thin-shell wormholes from regular Hayward $\mathrm{BH}$ and analyze their stability under linear perturbations. The surface energy density and surface pressure corresponding to static wormhole configuration at $a=a_{0}$ become

$$
\begin{aligned}
& \sigma_{0}=-\frac{\sqrt{F\left(a_{0}\right)}}{2 \pi a_{0}}, \\
& p_{0}=\frac{1}{8 \pi} \frac{2 F\left(a_{0}\right)+a_{0} F^{\prime}\left(a_{0}\right)}{a_{0} \sqrt{F\left(a_{0}\right)}} .
\end{aligned}
$$

The energy density and pressure follow the conservation identity $S_{; j}^{i j}=0$, which becomes for the line element (1) as follows:

$$
\frac{d}{d \tau}(\sigma \Phi)+p \frac{d \Phi}{d \tau}=0
$$

where $\Phi=4 \pi a^{2}$ corresponds to wormhole throat area. Using $\sigma^{\prime}=\dot{\sigma} / \dot{a}$, the above equation can be written as

$$
a \sigma^{\prime}=-2(\sigma+p) \text {. }
$$

The thin-shell equation of motion can be obtained by rearranging (6) as $\dot{a}^{2}+\Psi(a)=0$, which determines wormhole dynamics, while the potential function $\Psi(a)$ is defined by

$$
\Psi(a)=F(a)-[2 \pi a \sigma(a)]^{2} .
$$

In order to explore wormhole stability, we expand $\Psi(a)$ around $a=a_{0}$ using Taylor's series as

$$
\begin{aligned}
\Psi(a)= & \Psi\left(a_{0}\right)+\Psi\left(a_{0}\right)\left(a-a_{0}\right) \\
& +\frac{1}{2} \Psi^{\prime \prime}\left(a_{0}\right)\left(a-a_{0}\right)^{2}+O\left[\left(a-a_{0}\right)^{3}\right] .
\end{aligned}
$$

The first derivative of (18) through (17) takes the form

$$
\Psi^{\prime}(a)=F^{\prime}(a)+8 \pi^{2} a \sigma(a)[\sigma(a)+2 p(a)] .
$$

The stability of wormhole static solution depends upon $\Psi^{\prime \prime}\left(a_{0}\right) \gtrless 0$ and $\Psi^{\prime}\left(a_{0}\right)=0=\Psi\left(a_{0}\right)$. The surface stresses for static configuration (15) yield

$$
\begin{aligned}
& \sigma_{0}=-\frac{\sqrt{a_{0}^{3}+2 M l^{2}-2 M a_{0}^{2}}}{2 \pi a_{0} \sqrt{a_{0}^{3}+2 M l^{2}}}, \\
& p_{0}=\frac{a_{0}^{3}+2 M l^{2}-4 M a_{0}^{2}}{4 \pi a_{0} \sqrt{\left(a_{0}^{3}+2 M l^{2}\right)\left(a_{0}^{3}+2 M l^{2}-2 M a_{0}^{2}\right)}} .
\end{aligned}
$$

The choice of model for exotic matter has significant importance in the dynamical investigation of thin-shell wormholes. In a recent work, Halilsoy et al. [28] examined the dynamics of Hayward thin-shell wormholes for linear, logarithmic, and Chaplygin gas models. In this paper, we take VDW quintessence and GCCG and MCCG fluids at the shell to study stability of regular Hayward thin-shell wormholes. We will explore the possibility of the existence of stable traversable wormhole solutions by taking different EoS for exotic matter. In the following, we adopt standard stability formalism by the context of the above candidates of dark energy as exotic matter.

3.1. Van der Waals Quintessence. Firstly, we model the exotic matter by VDW quintessence EoS which is a remarkable scenario to describe accelerated expansion of the universe without the presence of exotic fluids. The EoS for VDW quintessence is given by

$$
p=\frac{\gamma \sigma}{1-B \sigma}-\alpha \sigma^{2}
$$


where $\alpha, B$, and $\gamma$ are EoS parameters. The specific values of these parameters lead to accelerated and decelerated periods. Inserting (15) in (22), the equation for static configuration is obtained as

$$
\begin{aligned}
& \left\{a_{0}^{2} \psi^{\prime}\left(a_{0}\right)+2 a_{0} \psi\left(a_{0}\right)+\frac{2 \alpha}{\pi}\left[\psi\left(a_{0}\right)\right]^{3 / 2}\right\} \\
& \cdot\left\{2 \pi^{2} a_{0}+B \pi \sqrt{\psi\left(a_{0}\right)}\right\}+2 \gamma\left(2 \pi a_{0}\right)^{2} \psi\left(a_{0}\right)=0 .
\end{aligned}
$$

The EoS turns out to be

$$
\begin{aligned}
& \sigma^{\prime}(a)+2 p^{\prime}(a)=\sigma^{\prime}(a)\left\{1+\frac{2}{1-B \sigma(a)}[\gamma-2 \alpha \sigma(a)\right. \\
& \left.\left.+B\left\{p(a)+3 \alpha \sigma^{2}(a)\right\}\right]\right\} .
\end{aligned}
$$

It is found that $\Psi(a)=\Psi^{\prime}(a)=0$ by substituting the values of $\sigma\left(a_{0}\right)$ and $p\left(a_{0}\right)$, while the second derivative of $\Psi$ through (20) and (24) becomes

$$
\begin{aligned}
& \Psi^{\prime \prime}\left(a_{0}\right)=F^{\prime \prime}\left(a_{0}\right)+\frac{\left[F^{\prime}\left(a_{0}\right)\right]^{2}}{2 F\left(a_{0}\right)}\left[\frac{B \sqrt{F\left(a_{0}\right)}}{2 \pi a_{0}+\sqrt{F\left(a_{0}\right)}}\right. \\
& -1]+\frac{F^{\prime}\left(a_{0}\right)}{a_{0}}\left[1+\frac{1}{2 \pi a_{0}+\sqrt{F\left(a_{0}\right)}}\left\{4 \pi a_{0} \gamma\right.\right. \\
& +4 \alpha \sqrt{F\left(a_{0}\right)} \\
& \left.\left.+B\left(2 \sqrt{F\left(a_{0}\right)}+\frac{3 \alpha B \sqrt{F\left(a_{0}\right)}}{\pi a_{0}}\right)\right\}\right] \\
& -\frac{2 F\left(a_{0}\right)}{a_{0}^{2}}(1+\gamma)\left[1+\frac{1}{2 \pi a_{0}+\sqrt{F\left(a_{0}\right)}}\left\{4 \pi a_{0} \gamma\right.\right. \\
& +4 \alpha \sqrt{F\left(a_{0}\right)} \\
& \left.\left.+B\left(2 \sqrt{F\left(a_{0}\right)}+\frac{3 \alpha B \sqrt{F\left(a_{0}\right)}}{\pi a_{0}}\right)\right\}\right] \text {. }
\end{aligned}
$$

Now, we formulate static solutions for which the dynamical equation through (23) takes the form

$$
\begin{aligned}
& 2 a_{0}^{4}\left(a_{0}^{3}+M l^{2}-M a_{0}^{2}\right)+8 M^{2} l^{2} a_{0}^{2}\left(1-2 a_{0}^{2}\right) \\
& +\frac{2 \alpha}{\pi}\left[2 \pi^{2} a_{0}\left(a_{0}^{3}+2 M l^{2}\right)^{1 / 2}\right. \\
& \cdot\left(a_{0}^{3}+M l^{2}-2 M a_{0}^{2}\right)^{3 / 2} \\
& \left.+B \pi\left(a_{0}^{3}+2 M l^{2}-2 M a_{0}^{2}\right)^{2}\right]+2 \gamma\left(2 \pi a_{0}^{2}\right)^{2}
\end{aligned}
$$

$$
\begin{aligned}
& \cdot\left[a_{0}^{3}\left(a_{0}^{3}+4 M l^{2}-2 M a_{0}^{2}\right)+4 M l^{2} a_{0}^{2}\left(a_{0}-1\right)\right] \\
& =0
\end{aligned}
$$

whose solutions correspond to static Hayward thin-shell wormholes. In order to explore the wormhole stability, we evaluate numerical value of throat radius $a_{0}$ (static) from (26) and substitute it into (25). We choose Hayward parameter values $l=0,0.1,0.7,0.9$ and check the role of increasing values of $l$ on the stability of Hayward thin-shell wormholes. We are interested to find the possibility of the existence of traversable thin-shell wormholes and to check whether the wormhole throat will expand or collapse under perturbation. For the existence of static stable solutions, $\Psi^{\prime \prime}>0$ and $a_{0}>r_{h}$, while $\Psi^{\prime \prime}<0$ and $a_{0}>r_{h}$ hold for unstable solutions. For $a_{0} \leq r_{h}$, no static solution exists leading to nonphysical region (grey zone). In this region, the stressenergy tensor may vanish leading to an event horizon which makes the wormhole no more traversable. The stable and unstable solutions correspond to green and yellow zones, respectively. The graphical results in Figures 3 and 4 can be summarized as follows.

For $\gamma \in(-\infty,-0.3]$, only unstable solutions exist corresponding to $l=0,0.1$, while both (stable and unstable) wormhole configurations appear by increasing the values of Hayward parameter; that is, $l=0.7,0.9$ as shown in Figure 3. For $l=0.7$, the wormhole is initially stable but its throat continues to expand leading to unstable solution. The nonphysical region in the wormhole configuration decreases gradually and vanishes for $l=0.9$. In this case, we find unstable wormhole solution for $a_{0}<2$ which leads to the collapse of wormhole throat as $B \alpha^{-(1+\gamma)}$ approaches its maximum value. For increasing $\gamma$, that is, $\gamma \in[0.1,0.9]$, there exist unstable and stable configurations.

Finally, we examine the stability of Hayward thin-shell wormholes for $\gamma \in[1, \infty)$ and find only stable solutions with $l=0,0.1,0.7$ which shows the expansion and traversability of wormhole throat. The unstable solution also appears for $l=0.9$ and $a_{0}<2$ leading to nontraversable wormhole due to its collapse. We find stable solutions for $a_{0}>2$ and the wormhole throat expands which allows the wormhole to open its mouth. Figure 4 shows the corresponding results for $\gamma=1$. This graphical analysis shows that the wormhole exhibits physical regions (stable/unstable) corresponding to different values of Hayward parameter. Since the regular Hayward wormholes are singularity-free due to their regular centers, the space-time has event horizons which give rise to nonphysical regions for $0<a_{0}<2.8$ and makes the wormhole nontraversable. We find that the wormhole can be made traversable as well as stable by tuning the Hayward parameter to its large value. Also, it is noted that $\gamma=1$ is the most fitted value to analyze only stable solutions.

3.2. Generalized Cosmic Chaplygin Gas. Now, we assume GCCG [30] to support the exotic matter at the shell. Chaplygin gas is a hypothetical substance that satisfies an exotic EoS. The EoS for GCCG is defined as

$$
p=-\frac{1}{\sigma^{\gamma}}\left[E+\left(\sigma^{1+\gamma}-E\right)^{-w}\right]
$$



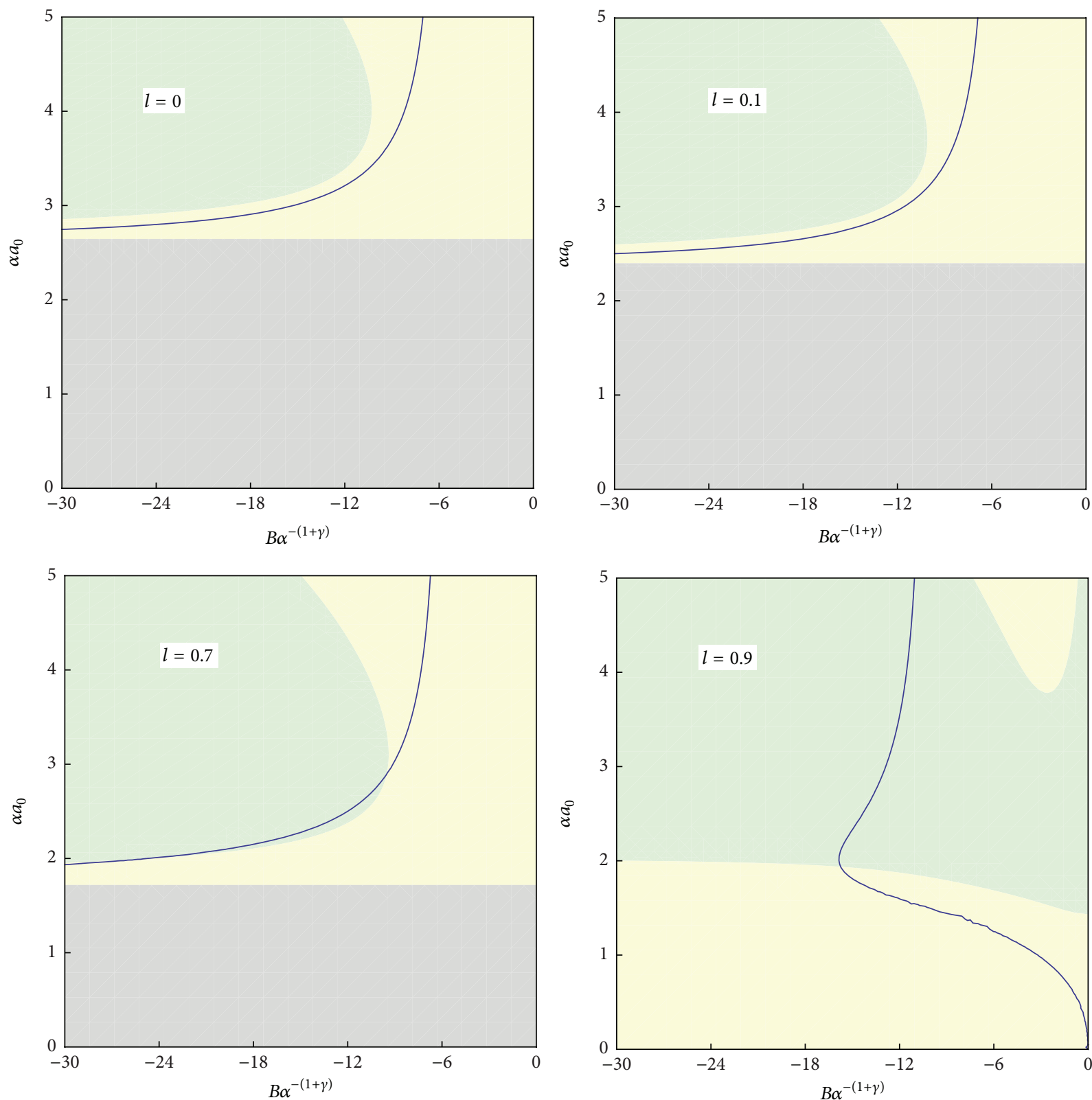

Figure 3: Plots for regular Hayward thin-shell wormholes by taking VDW quintessence with $\gamma=-0.5, M=1, \alpha=1$, and different values of Hayward parameter $l$. The stable and unstable regions are represented by green and yellow colors, respectively, while the grey zone corresponds to nonphysical region. Here, $B \alpha^{-(1+\gamma)}$ and $\alpha a_{0}$ are labeled along abscissa and ordinate, respectively.

where $E=B /(1+w)-1, B \in(-\infty, \infty),-C<w<0$, and $C$ is a positive constant rather than unity. The dynamical equation for static solutions through (15) and (27) yields

$$
\begin{aligned}
{\left[a_{0}^{2} F^{\prime}\left(a_{0}\right)+2 a_{0} F\left(a_{0}\right)\right]\left[2 a_{0}\right]^{\gamma}-2\left(4 \pi a_{0}^{2}\right)^{1+\gamma} } & \cdot\left[F\left(a_{0}\right)\right]^{(1-\gamma) / 2} \\
\cdot & {\left[E+\left\{\left(2 \pi a_{0}\right)^{-(1+\gamma)}\left(F\left(a_{0}\right)\right)^{(1+\gamma) / 2}-E\right\}^{-w}\right]=0 . }
\end{aligned}
$$

Differentiation of (27) with respect to $a$ leads to

$$
\begin{aligned}
& \sigma^{\prime}(a)+2 p^{\prime}(a)=\sigma^{\prime}(a) \\
& \cdot\left[1+2 w(1+\gamma)\left\{\sigma^{1+\gamma}-E\right\}^{-(1+w)}-\frac{2 \gamma p(a)}{\sigma(a)}\right],
\end{aligned}
$$

which determines the second derivative of potential function as

$$
\Psi^{\prime \prime}\left(a_{0}\right)=F^{\prime \prime}\left(a_{0}\right)+\frac{(\gamma-1)\left[F^{\prime}\left(a_{0}\right)\right]^{2}}{2 F\left(a_{0}\right)}+\frac{F^{\prime}\left(a_{0}\right)}{a_{0}}[1
$$

$$
\left.+2 w(1+\gamma)\left\{\left(\frac{\sqrt{F\left(a_{0}\right)}}{2 \pi a_{0}}\right)^{1+\gamma}-E\right\}^{-(1+w)}\right]
$$



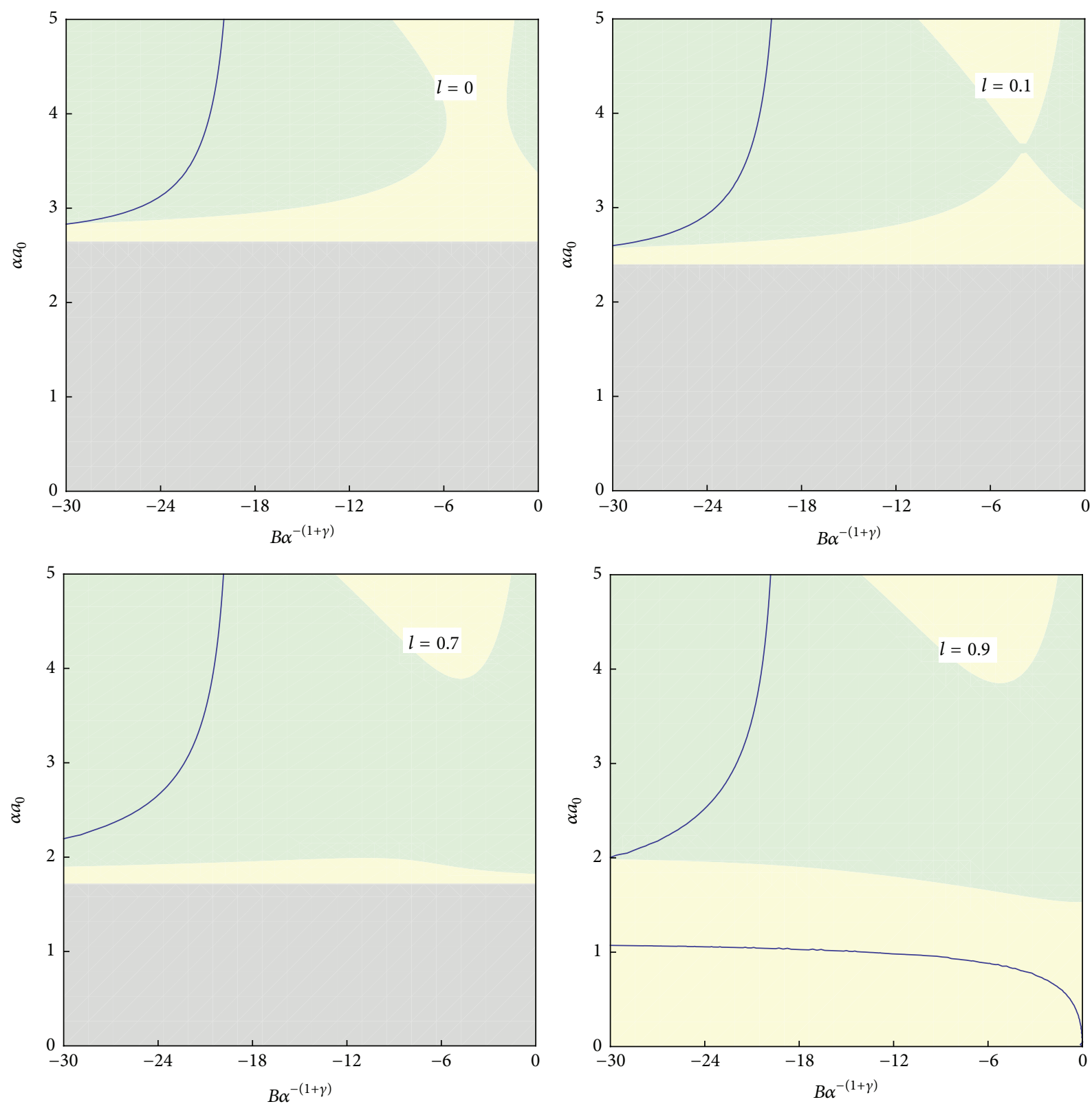

FIgUre 4: Plots for VDW quintessence EoS taking $\gamma=1, M=1, \alpha=1$, and different values of Hayward parameter $l$.

$$
\begin{aligned}
& -\frac{2 F\left(a_{0}\right)}{a_{0}^{2}}(1+\gamma)[1 \\
& \left.+2 w\left\{\left(\frac{\sqrt{F\left(a_{0}\right)}}{2 \pi a_{0}}\right)^{1+\gamma}-E\right\}^{-(1+w)}\right] .
\end{aligned}
$$

Using (21) in (28), the corresponding dynamical equation for static solution becomes

$$
\begin{aligned}
a_{0}^{3} & +2 M l^{2}-4 M a_{0}^{2}-2\left(2 \pi a_{0}\right)^{1+\gamma}\left[a_{0}^{3}+2 M l^{2}\right. \\
- & \left.2 M a_{0}^{2}\right]^{(1-\gamma) / 2}\left[E+\left\{\left(2 \pi a_{0}\right)^{-(1+\gamma)}\right.\right.
\end{aligned}
$$

$$
\begin{aligned}
& \cdot\left(a_{0}^{3}+2 M l^{2}\right)^{-(1+\gamma) / 2}\left(a_{0}^{3}+2 M l^{2}-2 M a_{0}^{2}\right)^{(1+\gamma) / 2} \\
& \left.-E\}^{-w}\right]=0
\end{aligned}
$$

which gives static regular Hayward wormhole solutions. Here, we again employ the same technique for the stability analysis as in the previous subsection. The results in Figures 5 and 6 correspond to GCCG. For $\gamma=0.2,1$ and $l=$ $0,0.1,0.7$, we find fluctuating wormhole solutions. It is found that unstable solution exists for small values of throat radius $a_{0}$, while the solutions become stable when the throat radius expands. There exists a nonphysical region for $0<a_{0}<2$ which diminishes with $l=0.9$ making a stable traversable 

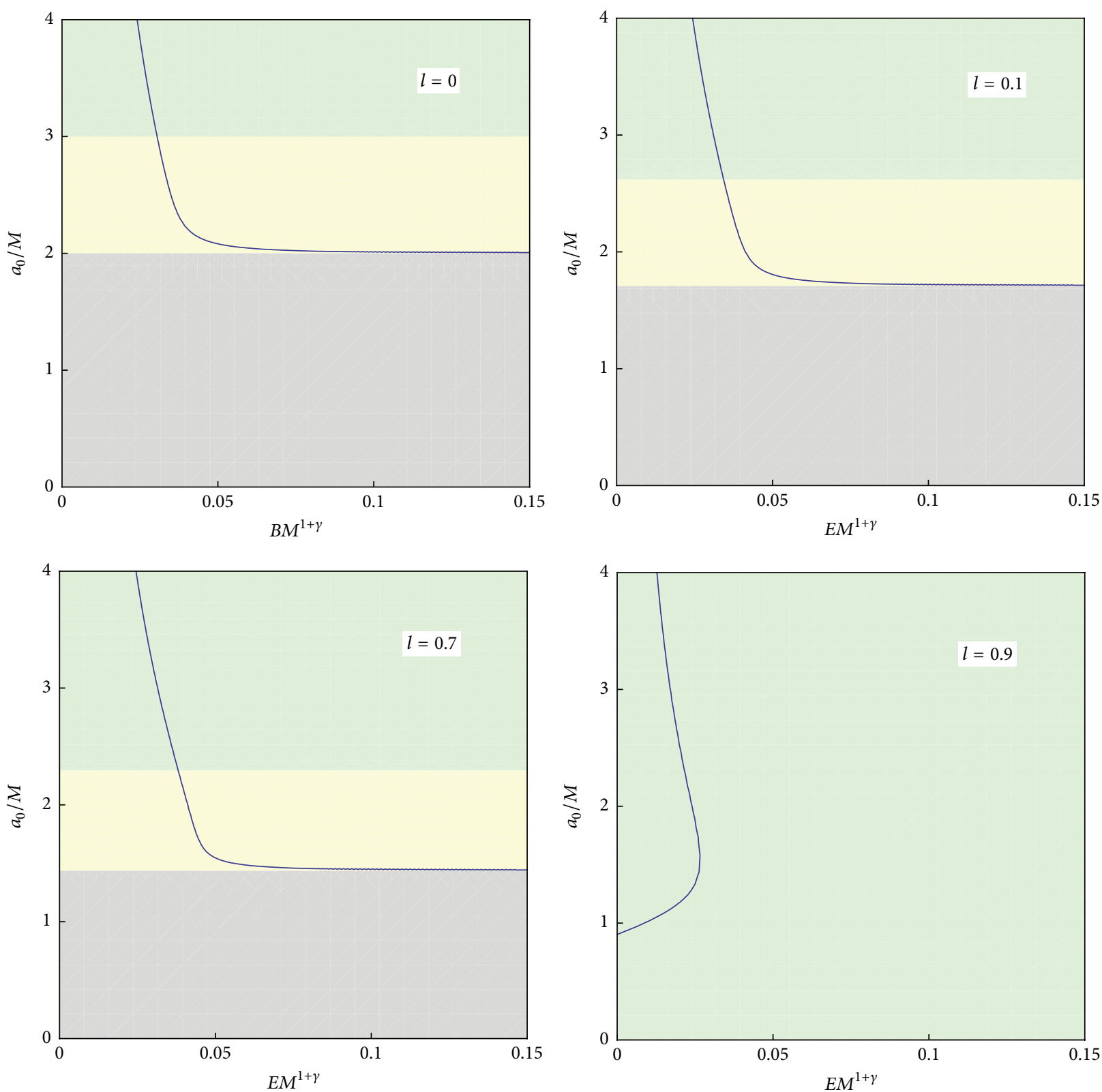

Figure 5: Plots for GCCG with parameters $\gamma=0.2, M=1$, and $w=-10$ and different values of $l$. Here, $E M^{(1+\gamma)}$ and $a_{0} / M$ are labeled along abscissa and ordinate, respectively.

wormhole. For $\gamma=1$ and $l=0.9$, there is a fluctuating behavior of wormhole throat. Initially, it is stable for smaller values of $a_{0}$ but becomes unstable by increasing throat radius. Finally, we analyze stable regular Hayward thinshell wormhole for throat radius $a_{0}>2$ which undergoes expansion.

3.3. Modified Cosmic Chaplygin Gas. Here, we assume MCCG model for exotic matter for which EoS is given by

$$
p=A \sigma-\frac{1}{\sigma^{\gamma}}\left[E+\left(\sigma^{1+\gamma}-E\right)^{-w}\right] .
$$

Sadeghi and Farahani [31] assumed MCCG as varying by considering $E$ as a function of scale factor $a$, while, in our case, $E$ is assumed as a constant. Substituting (15) in (32), the dynamical equation (for static configuration) is

$$
\begin{aligned}
& {\left[a_{0}^{2} F^{\prime}\left(a_{0}\right)+2 a_{0} F\left(a_{0}\right)\right](1+2 A)\left[2 a_{0}\right]^{\gamma}} \\
& \quad-2\left(4 \pi a_{0}^{2}\right)^{1+\gamma}\left[F\left(a_{0}\right)\right]^{(1-\gamma) / 2} \\
& \quad \cdot\left[E+\left\{\left(2 \pi a_{0}\right)^{-(1+\gamma)}\left(F\left(a_{0}\right)\right)^{(1+\gamma) / 2}-E\right\}^{-w}\right]=0 .
\end{aligned}
$$

The first derivative of EoS with respect to $a$ yields

$$
\begin{aligned}
& \sigma^{\prime}(a)+2 p^{\prime}(a)=\sigma^{\prime}(a)[1 \\
& \left.\quad+2(1+\gamma)\left\{A+w\left(\sigma^{1+\gamma}-E\right)^{-(1+w)}\right\}-\frac{2 \gamma p(a)}{\sigma(a)}\right] .
\end{aligned}
$$



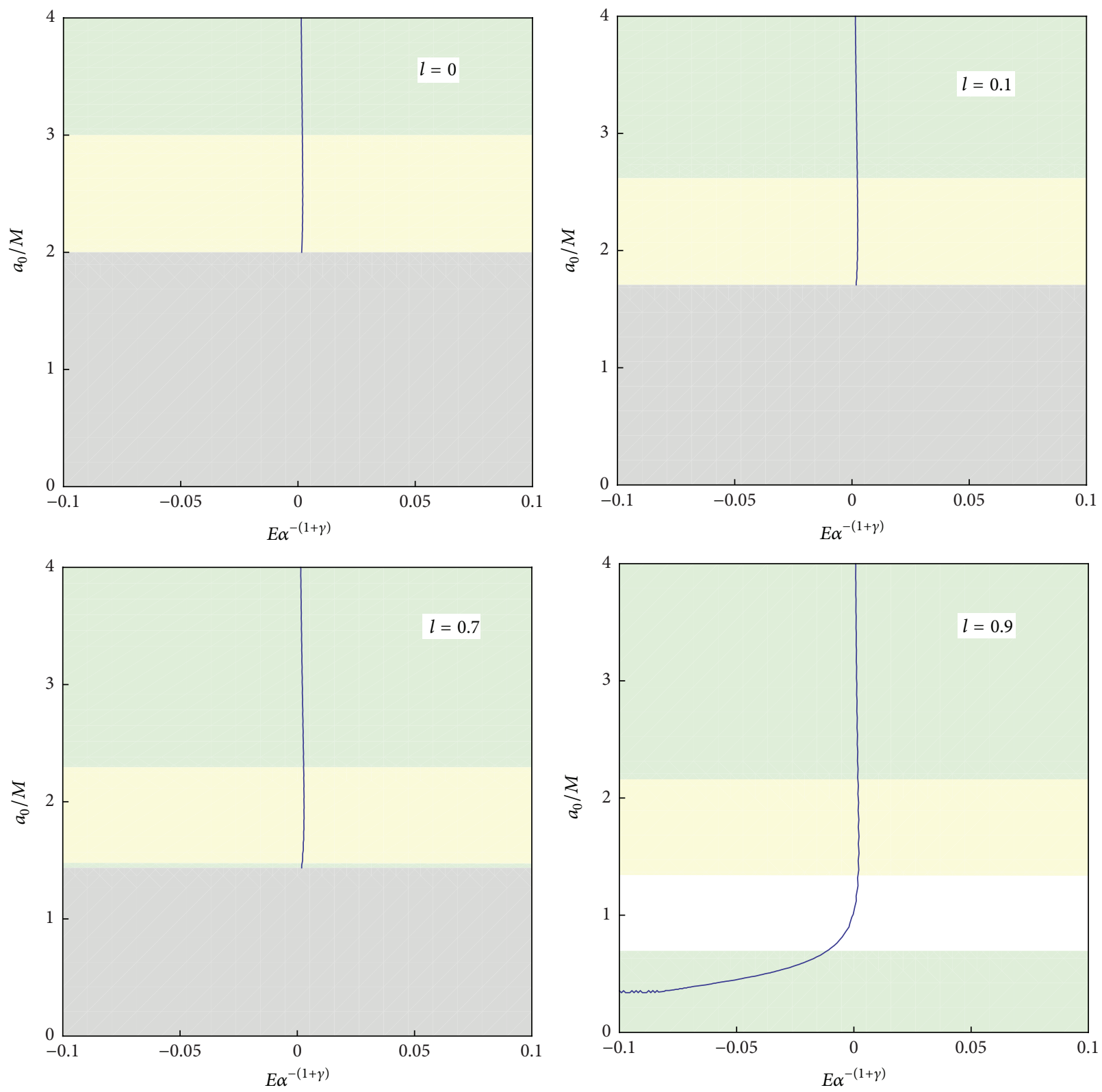

Figure 6: Plots for GCCG with $\gamma=1, M=1, w=-10$, and different values of $l$.

It is noted that $\Psi(a)=\Psi^{\prime}(a)=0$ at $a=a_{0}$, while the second derivative of $\Psi(a)$ through (34) takes the form

$$
\begin{aligned}
& \Psi^{\prime \prime}\left(a_{0}\right)=F^{\prime \prime}\left(a_{0}\right)+\frac{(\gamma-1)\left[F^{\prime}\left(a_{0}\right)\right]^{2}}{2 F\left(a_{0}\right)}+\frac{F^{\prime}\left(a_{0}\right)}{a_{0}}[1 \\
& +2\{A \\
& \left.\left.+w(1+\gamma)\left\{\left(\frac{\sqrt{F\left(a_{0}\right)}}{2 \pi a_{0}}\right)^{1+\gamma}-E\right\}^{-(1+w)}\right\}\right]
\end{aligned}
$$

$$
\begin{aligned}
& -\frac{2 F\left(a_{0}\right)}{a_{0}^{2}}(1+\gamma)[1+2(1+\gamma)[A \\
& \left.\left.+w\left\{\left(\frac{\sqrt{F\left(a_{0}\right)}}{2 \pi a_{0}}\right)^{1+\gamma}-E\right\}^{-(1+w)}\right]\right] .
\end{aligned}
$$

For Hayward wormhole static solutions, (33) turns out to be

$$
\begin{gathered}
a_{0}^{3}\left(a_{0}^{3}+M l^{2}-4 M a_{0}^{2}\right)+4 M^{2} l^{2}\left(l^{2}-4 a_{0}^{2}\right)+2 A \\
-\left(2 \pi a_{0}\right)^{1+\gamma}\left(a_{0}^{3}+2 M l^{2}\right)^{(3+\gamma) / 2}\left(a_{0}^{3}+2 M l^{2}\right.
\end{gathered}
$$



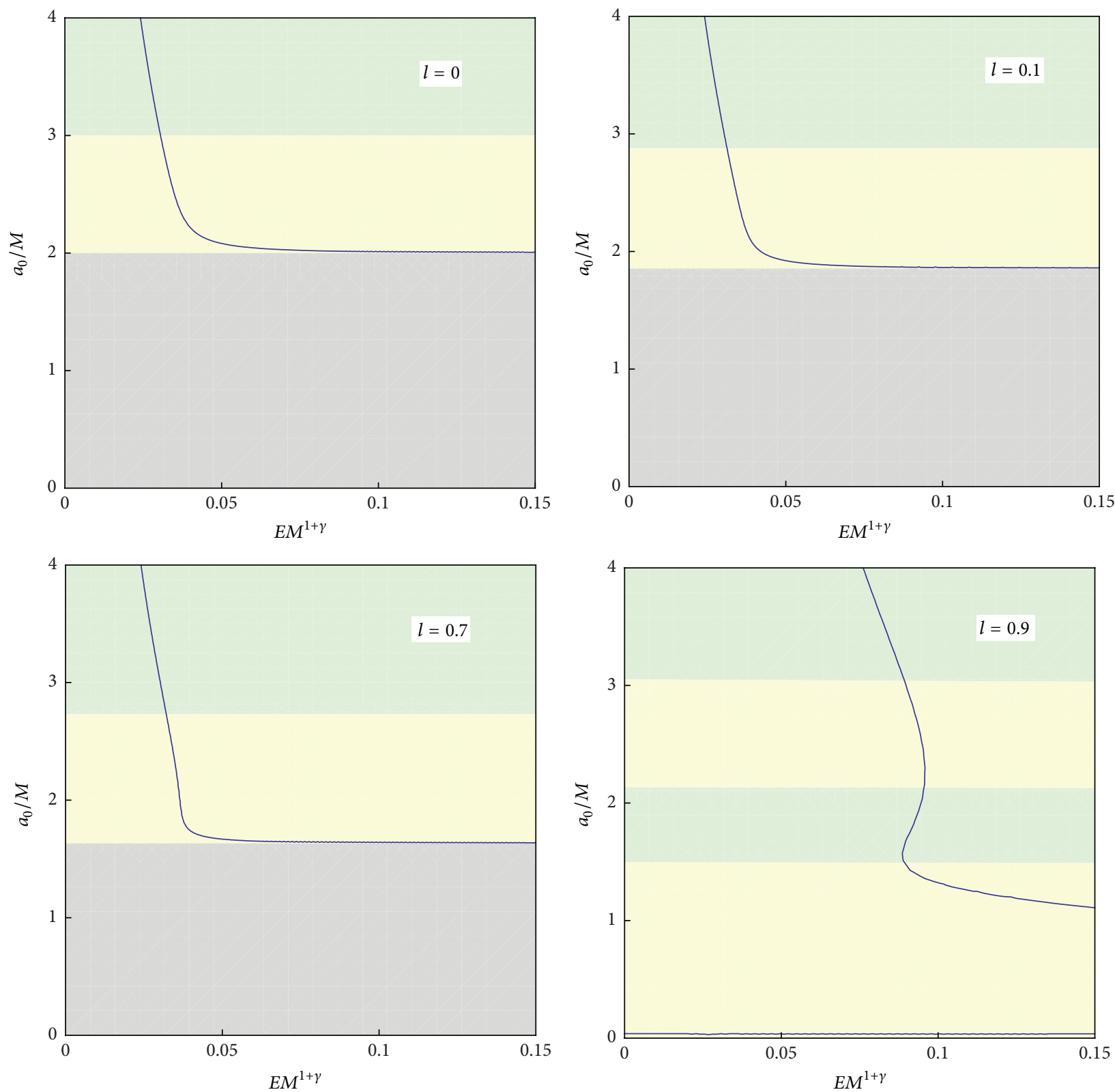

Figure 7: Plots for MCCG with $\gamma=0.2, M=1, A=1$, and $w=-10$ with different values of $l$. Here, $E M^{(1+\gamma)}$ and $a_{0} / M$ are labeled along abscissa and ordinate, respectively.

$$
\begin{aligned}
& \left.-2 M a_{0}^{2}\right)^{-(1+\gamma) / 2}\left[E+\left\{\left(2 \pi a_{0}\right)^{-(1+\gamma)}\right.\right. \\
& \cdot\left(a_{0}^{3}+2 M l^{2}\right)^{-(1+\gamma) / 2}\left(a_{0}^{3}+2 M l^{2}-2 M a_{0}^{2}\right)^{(1+\gamma) / 2} \\
& \left.-E\}^{-w}\right]=0 .
\end{aligned}
$$

and eventually vanishes for $l=0.9$ making a traversable wormhole. For $l=0.9$, we find a traversable wormhole solution with fluctuating behavior of wormhole throat which shows a stable wormhole solution with throat expansion. It is noted that the stability region increases by increasing values of $l$.

The results in Figures 7 and 8 show that, for $l=0,0.1,0.7$, one stable solution and one unstable solution exist for $\gamma=0.2,0.6$. We analyze that two stable and two unstable regions appear for $l=0.9$. There exists a nonphysical region for $0<a_{0}<2$ again showing the event horizon which continues to decrease

\section{Concluding Remarks}

In this paper, we have constructed regular Hayward thinshell wormholes by implementing Visser's cut-and-paste technique and analyzed their stability by incorporating the effects of increasing values of Hayward parameter. The 

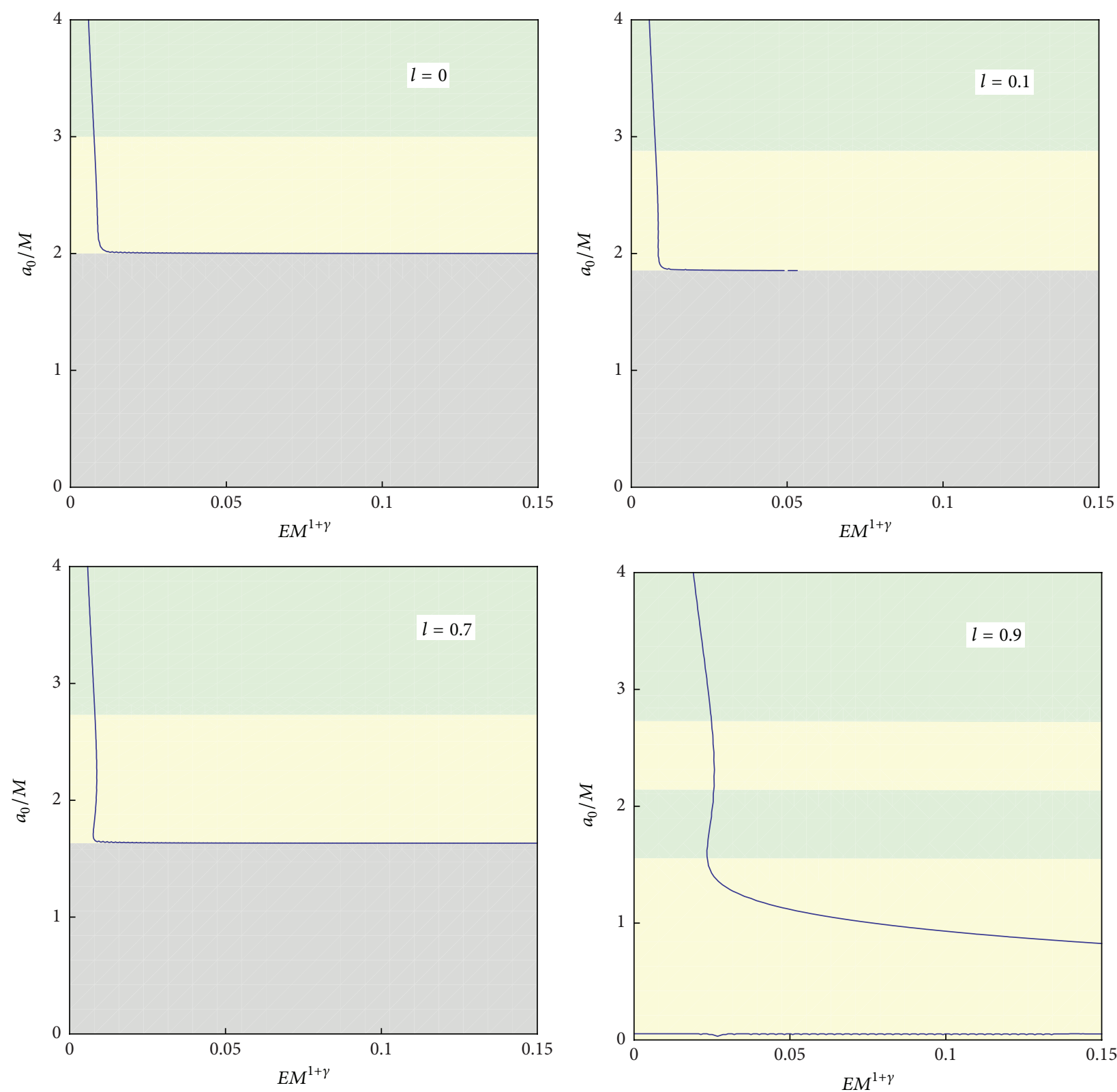

FigURE 8: Plots corresponding to MCCG with parameters $\gamma=0.6, M=1, A=1$, and $w=-10$ and different values of $l$.

surface stresses have been found by using Lanczos equations. The sum of surface stresses of matter indicates the violation of NEC which is a fundamental ingredient in wormhole physics leading to the existence of exotic matter. It is found that the wormhole has attractive and repulsive characteristics corresponding to $a^{r}>0$ and $a^{r}<0$, respectively. For a convenient trip through a wormhole, an observer should not be dragged away by enormous tidal forces which requires that the acceleration felt by the observer must not exceed Earth's acceleration. The construction of viable thin-shell wormholes depends on total amount of exotic matter confined within the shell. Here, we have quantified the total amount of exotic matter by the volume integral theorem which is consistent with the fact that a small quantity of exotic matter is needed to support the wormhole. We have obtained a dynamical equation which determines possible throat radii for the static wormhole configurations.

It is found that one may construct a traversable wormhole theoretically with arbitrarily small amount of fluid describing cosmic expansion. In this context, we have analyzed stability of the regular Hayward thin-shell wormholes by taking VDW quintessence and GCCG and MCCG models at the wormhole throat. Firstly, we have investigated the possibility of stable traversable wormhole solutions using VDW quintessence EoS which describes cosmic expansion without the presence of exotic fluids. We have analyzed both stable and unstable wormhole configurations for small values of EoS parameter $\gamma$. The graphical analysis shows a nonphysical region (grey 
zone) for $0<a_{0}<2.8$ (Figures 3 and 4 ). In this region, the stress-energy tensor may vanish leading to an event horizon and the wormhole becomes nontraversable producing a black hole. The nonphysical region in the wormhole configuration decreases gradually and vanishes for $l=0.9$. In this case, we find unstable wormhole solution as $B \alpha^{-(1+\gamma)}$ approaches its maximum value. We have also examined stability of Hayward thin-shell wormholes for $\gamma \in[1, \infty)$ and found only stable solutions with $l=0,0.1,0.7$ which show expansion of the wormhole throat. It is worth mentioning here that regular Hayward thin-shell wormholes can be made traversable as well as stable by tuning the Hayward parameter to its large value. Also, it is noted that VDW quintessence fluid minimizes the usage of exotic matter and $\gamma=1$ is the best fitted value which induces only stable solutions.

In case of GCCG, we have analyzed fluctuating (stable and unstable) solutions for $l=0,0.1,0.7$ and $\gamma=0.2,1$. It is found that unstable solution exists for small values of throat radius $a_{0}$, which becomes stable when the throat radius expands. There exists a nonphysical region for $0<a_{0}<2$ which diminishes for $l=0.9$ making a stable traversable wormhole. For $\gamma=1$, we have analyzed stable regular Hayward thin-shell wormhole for throat radius $a_{0}>2$ which undergoes expansion. We have found that only stable wormhole configurations exist for the Hayward parameter $l=0.9$. Finally, for MCCG, we have found one stable and one unstable region for $l=0,0.1,0.7$, while these regions become double (two stable and two unstable) for $l=0.9$ (Figures 7 and 8). There exists a nonphysical region for $0<$ $a_{0}<2$ again showing the event horizon which eventually vanishes for $l=0.9$ making a traversable wormhole. It is noted that the stability region increases by increasing values of $l$. It was found that small radial perturbations yield no stable solutions for the regular Hayward thin-shell wormholes [28]. We conclude that stable regular Hayward wormhole solutions are possible against radial perturbations for VDW quintessence and GCCG and MCCG models. The trivial case $l=0$ corresponds to the Schwarzschild thinshell wormhole. It is worth mentioning here that the Hayward parameter increases stable regions for regular Hayward thinshell wormholes.

\section{Competing Interests}

The authors declare that they have no competing interests regarding the publication of this paper.

\section{References}

[1] C. W. Misner and J. A. Wheeler, "Classical physics as geometry," Annals of Physics, vol. 2, no. 6, pp. 525-603, 1957.

[2] S. A. Hayward, "Black holes and traversible wormholes: a synthesis," http://arxiv.org/abs/gr-qc/0203051.

[3] M. S. Morris and K. S. Thorne, "Wormholes in spacetime and their use for interstellar travel: a tool for teaching general relativity," American Journal of Physics, vol. 56, no. 5, pp. 395412, 1988.
[4] J. P. Lemos, “Three-dimensional black holes and cylindrical general relativity," Physics Letters B, vol. 353, no. 1, pp. 46-51, 1995.

[5] W. Israel, "Singular hypersurfaces and thin shells in general relativity," Il Nuovo Cimento B Series 10, vol. 48, no. 2, p. 463, 1967.

[6] K. K. Nandi, Y.-Z. Zhang, and K. B. V. Kumar, "Volume integral theorem for exotic matter," Physical Review D, vol. 70, no. 12, Article ID 127503, 2004.

[7] M. Visser, "Traversable wormholes: some simple examples," Physical Review D, vol. 39, no. 10, pp. 3182-3184, 1989.

[8] M. Visser, Lorentzian Wormholes, AIP Series in Computational and Applied Mathematical Physics, AIP Press, 1996.

[9] S. W. Kim and H. Lee, "Exact solutions of a charged wormhole," Physical Review D, vol. 63, no. 6, Article ID 064014, 2001.

[10] M. Ishak and K. Lake, "Stability of transparent spherically symmetric thin shells and wormholes," Physical Review D, vol. 65, no. 4, Article ID 044011, 2002.

[11] F. S. N. Lobo and P. Crawford, "Linearized stability analysis of thin-shell wormholes with a cosmological constant," Classical and Quantum Gravity, vol. 21, no. 2, pp. 391-404, 2004.

[12] E. F. Eiroa and G. E. Romero, "Linearized stability of charged thin-shell wormholes," General Relativity and Gravitation, vol. 36, no. 4, pp. 651-659, 2004.

[13] M. Sharif and M. Azam, "Spherical thin-shell wormholes and modified Chaplygin gas," Journal of Cosmology and Astroparticle Physics, vol. 2013, no. 5, p. 25, 2013.

[14] M. Sharif and S. Mumtaz, "Stability of thin-shell wormholes from regular abg black hole," https://arxiv.org/abs/1604.01012.

[15] M. Sharif and S. Mumtaz, "Influence of nonlinear electrodynamics on stability of thin-shell wormholes," Astrophysics and Space Science, vol. 361, no. 7, article 218, 2016.

[16] A. Das and S. Kar, "The Ellis wormhole with 'tachyon matter," Classical and Quantum Gravity, vol. 22, no. 14, pp. 3045-3053, 2005.

[17] F. S. N. Lobo, "Chaplygin traversable wormholes," Physical Review D, vol. 73, Article ID 064028, 2006.

[18] E. F. Eiroa and C. Simeone, "Stability of Chaplygin gas thin-shell wormholes," Physical Review D, vol. 76, no. 2, Article ID 024021, 2007.

[19] F. S. N. Lobo, "Thin-shell wormholes with a generalized Chaplygin gas," Physical Review D, vol. 80, Article ID 044033, 2009.

[20] S. Capozziello, V. F. Cardone, S. Carloni et al., "Constraining Van der Waals quintessence with observations," Journal of Cosmology and Astroparticle Physics, vol. 2005, no. 4, p. 5, 2005.

[21] E. F. Eiroa, "Thin-shell wormholes with a generalized Chaplygin gas," Physical Review D, vol. 80, no. 4, Article ID 044033, 2009.

[22] P. K. F. Kuhfittig, "The stability of thin-shell wormholes with a phantom-like equation of state," Acta Physica Polonica B, vol. 41, no. 9, pp. 2017-2029, 2010.

[23] M. Sharif and M. Azam, "Reissner-Nordström thin-shell wormholes with generalized cosmic Chaplygin gas," The European Physical Journal C, vol. 73, no. 9, article 2554, 2013.

[24] M. Sharif and S. Mumtaz, "Schwarzschild-de sitter and anti-de sitter thin-shell wormholes and their stability," Advances in High Energy Physics, vol. 2014, Article ID 639759, 13 pages, 2014.

[25] F. S. N. Lobo, "Van der Waals quintessence stars," Physical Review D, vol. 75, no. 2, Article ID 024023, 2007.

[26] M. Sharif and S. Mumtaz, "Effects of charge on the stability of thin-shell wormholes," Astrophysics and Space Science, vol. 352, no. 2, pp. 729-736, 2014. 
[27] M. Sharif and S. Mumtaz, "Linearized stability of cylindrical thin-shell wormholes," Canadian Journal of Physics, vol. 94, no. 2, pp. 158-169, 2016.

[28] M. Halilsoy, A. Ovgun, and S. H. Mazharimousavi, “Thin-shell wormholes from the regular Hayward black hole," European Physical Journal C, vol. 74, no. 3, pp. 1-7, 2014.

[29] S. A. Hayward, "Formation and evaporation of nonsingular black holes," Physical Review Letters, vol. 96, Article ID 031103, 2006.

[30] P. F. Gonzalez-Diaz, "You need not be afraid of phantom energy," Physical Review D, vol. 68, no. 2, Article ID 021303, 2003.

[31] J. Sadeghi and H. Farahani, "Interaction between viscous varying modified cosmic Chaplygin gas and Tachyonic fluid," Astrophysics and Space Science, vol. 347, no. 1, pp. 209-219, 2013. 

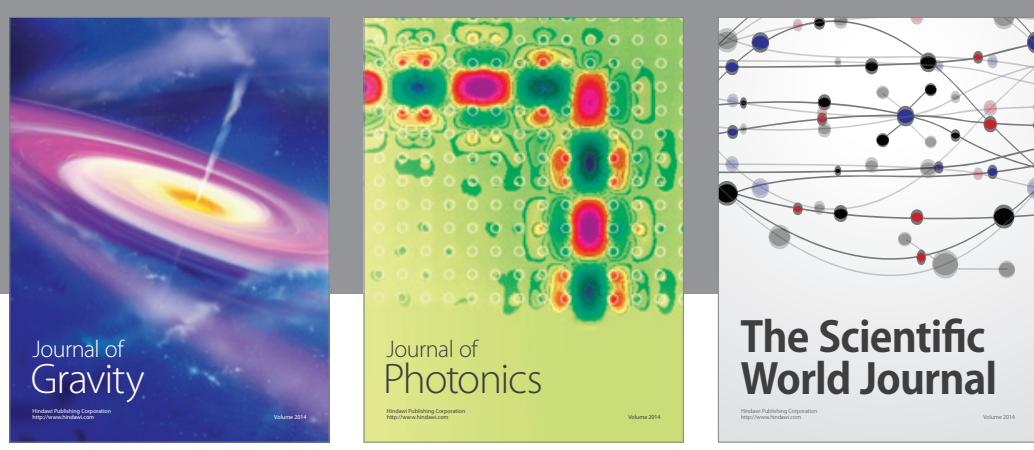

The Scientific World Journal
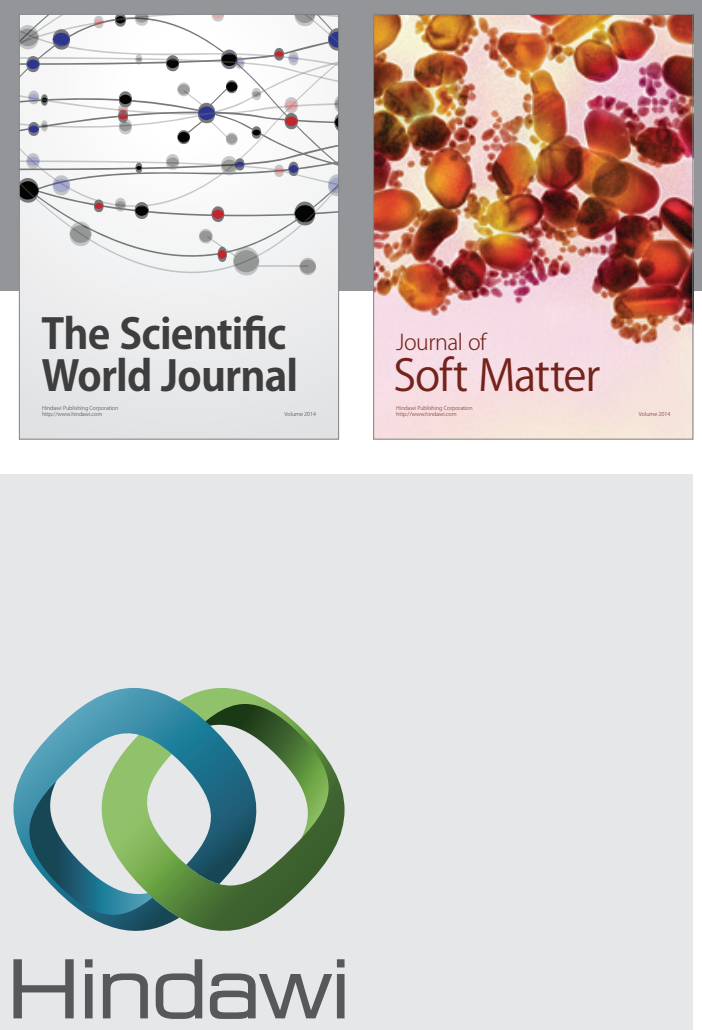

Submit your manuscripts at

http://www.hindawi.com

nternational Journal of

Statistical Mechanics
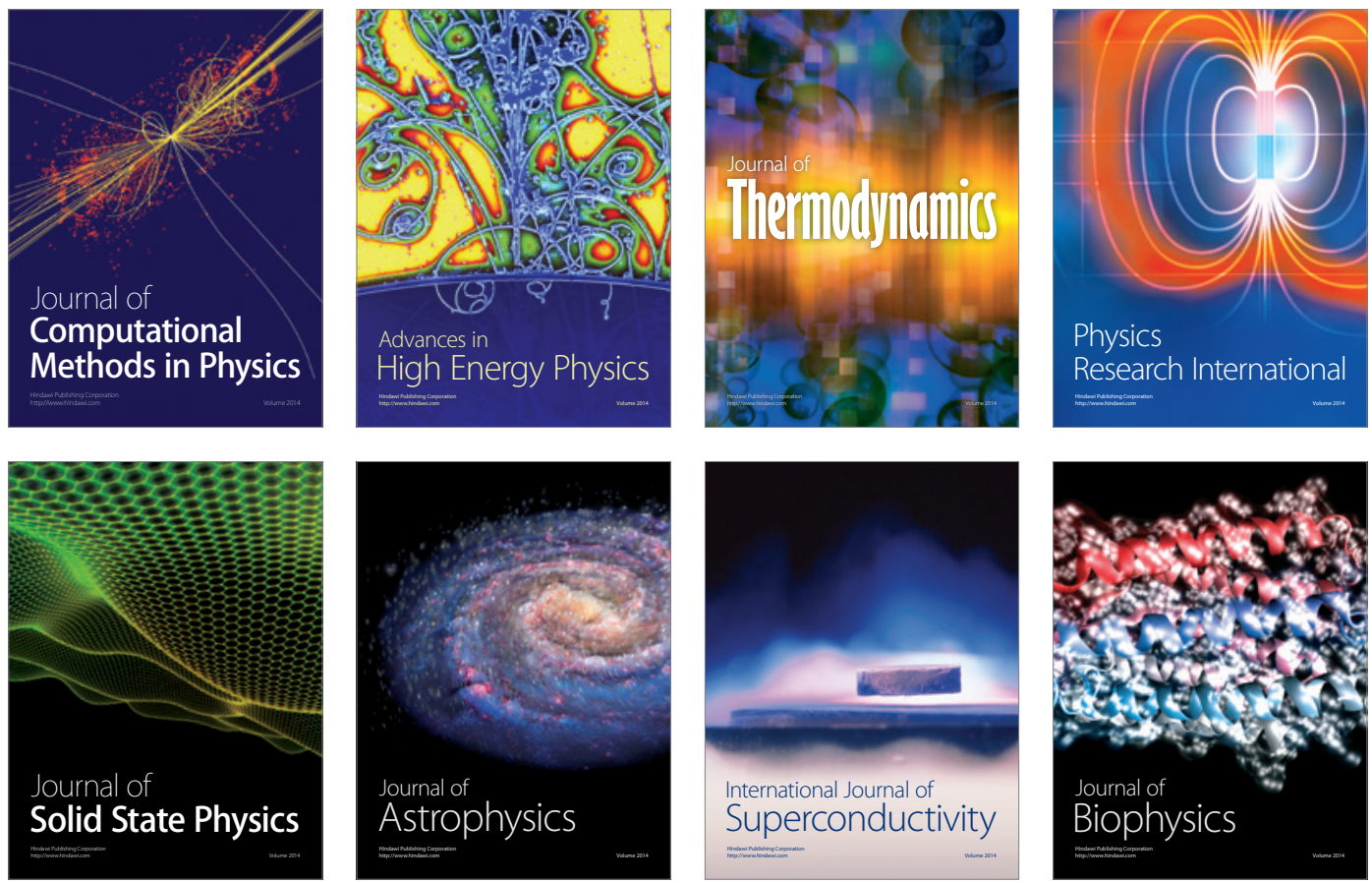
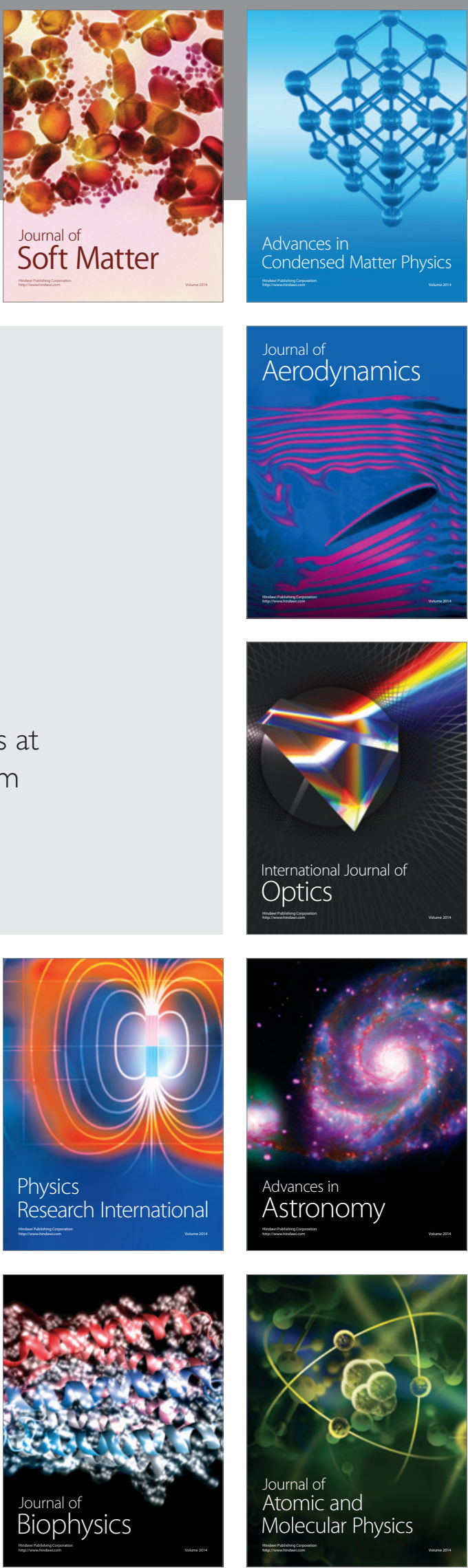